\title{
Epigenetics and Depression: An Update
}

\author{
Eugene Lin ${ }^{1,2,3}$ and Shih-Jen Tsai ${ }^{4,5,6} \bowtie$ \\ ${ }^{1}$ Department of Biostatistics, University of Washington, Seattle, WA, USA \\ ${ }^{2}$ Department of Electrical \& Computer Engineering, University of Washington, Seattle, WA, USA \\ ${ }^{3}$ Graduate Institute of Biomedical Sciences, China Medical University, Taichung, Taiwan \\ ${ }^{4}$ Department of Psychiatry, Taipei Veterans General Hospital, Taipei, Taiwan \\ ${ }^{5}$ Division of Psychiatry, National Yang-Ming University, Taipei, Taiwan \\ ${ }^{6}$ Institute of Brain Science, National Yang-Ming University, Taipei, Taiwan
}

Objective Depression is associated with various environmental risk factors such as stress, childhood maltreatment experiences, and stressful life events. Current approaches to assess the pathophysiology of depression, such as epigenetics and gene-environment (GxE) interactions, have been widely leveraged to determine plausible markers, genes, and variants for the risk of developing depression.

Methods We focus on the most recent developments for genomic research in epigenetics and GxE interactions.

Results In this review, we first survey a variety of association studies regarding depression with consideration of GxE interactions. We then illustrate evidence of epigenetic mechanisms such as DNA methylation, microRNAs, and histone modifications to influence depression in terms of animal models and human studies. Finally, we highlight their limitations and future directions.

Conclusion In light of emerging technologies in artificial intelligence and machine learning, future research in epigenetics and GxE interactions promises to achieve novel innovations that may lead to disease prevention and future potential therapeutic treatments for depression.

Psychiatry Investig 2019;16(9):654-661

Key Words Depression, Biomarkers, Epigenetics, Gene-environment interactions, Stress.

\section{INTRODUCTION}

Depression is one of the most prevalent, heterogeneous, and complicated psychiatric syndromes worldwide. ${ }^{1,2}$ Genomewide association studies (GWASs) and meta-analysis GWAS studies have identified more and more genes and single nucleotide polymorphisms (SNPs) associated with depression. ${ }^{3-5}$ For instance, a recent meta-analysis study $(\mathrm{n}=807,553)$ of GWASs pinpointed 87 significant SNPs after multiple testing correction and revealed pathways relating to neurotransmission and response to stimuli by employing an independent replication sample $(n=1,507,153){ }^{6}$ It has been suggested that epigenetic and environmental mechanisms play a substantial role for the pathophysiology of depression, where epigenetic

Received: June 24, 2019 Accepted: July 17, 2019

$\triangle$ Correspondence: Shih-Jen Tsai, MD

Department of Psychiatry, Taipei Veterans General Hospital, No. 201, Shih-Pai Road, Sec. 2, 11217, Taipei, Taiwan

Tel: +886-2-2875-7027, Fax: +886-2-2872-5643

E-mail: tsai610913@gmail.com

(c) This is an Open Access article distributed under the terms of the Creative Commons Attribution Non-Commercial License (https://creativecommons.org/licenses/bync/4.0) which permits unrestricted non-commercial use, distribution, and reproduction in any medium, provided the original work is properly cited. factors bridge the environmental and genetic mechanisms. Numerous investigations have utilized state-of-the-art methods such as epigenetics and gene-environment $(\mathrm{GxE})$ interactions to determine whether specific genes are involved with the pathophysiology of depression in human and animal studies of genomic applications. As a consequence, accumulating evidence indicates that an interplay between epigenetic/environmental mechanisms and applicable genes is certainly linked to patients with depression when compared with healthy controls. ${ }^{7.8}$ While a mammoth amount of plausible genetic markers in epigenetics and GxE interactions have been explored to be linked to depression, a further and more intensive exploration in support of this hypothesis is still warranted. ${ }^{1,9}$ In this review, we in brief summarize a variety of existing human and animal studies with consideration of epigenetics and GxE interactions in assessing and understanding putative epigenetic and environmental mechanisms with an increased risk of developing depression.

In this review, we first survey the candidate variant and genes that have been investigated as probable genetic markers and have been shown to be associated with depression in various studies of $\mathrm{GxE}$ interactions. Moreover, we assess vari- 
ous probable variant and genes that have been pinpointed in various epigenetic studies and have been discovered to be associated with depression. Finally, we show the limitations and future perspectives with respect to the previous studies in epigenetics and GxE interactions. In order to establish the roles of the probable variant and genes discovered in the previous human and animal studies, future studies with replication sample in extensive and independent cohorts will be warranted in terms of epigenetics and GxE interactions for depression.

\section{METHODS}

\section{Literature search and analyses}

In this review, we present relevant studies on epigenetics and GxE interactions in depression after a comprehensive search of the electronic PubMed database (2010-present). Key words in the search included "epigenetics," "depression," "methylation," "MicroRNAs," "Histone modifications," "GxE interactions," and "stress." Furthermore, we employed a manual search procedure of bibliographical cross-referencing. We manually screened the obtained articles and aimed at identifying original papers and reviews with a particular focus on epigenetics and $\mathrm{GxE}$ interactions in depression research. While this article is by no means a comprehensive review of all potential studies reported in the literature, we merely pinpointed various examples for epigenetics and GxE interactions in depression studies.

\section{RESULTS}

\section{Environmental factors on depression}

Environmental risk factors for depression have been a focus of attention in the literature and encompass stress, various abuse (such as physical, emotional, and sexual abuse), early adverse childhood experiences (such as childhood maltreatment and neglect), and stressful life events (such as marriage and family conflicts, adverse conditions during perinatal stage, disrupted interpersonal relationships, crime and legal issues, loss events, job-related concerns, financial hardship, and events related to unpleasant health problems). ${ }^{10}$

\section{GxE interactions on depression}

Here we target various association studies that have assessed potential interactions between environmental risk factors and biomarkers to verify the hypothesis that the proposed candidate variants and genes may contribute to depression via complex GxE interactions. Various loci such as CRHR1, FKBP5, $H T R 2 A$, and serotonin transporter gene-linked polymorphic region (5-HTTLPR) in the SLC6A4 (serotonin neurotransmit- ter transporter) gene have been robustly replicated and found to be linked to depression-relevant GxE interactions. ${ }^{11}$ This review is not intended as a comprehensive survey of all related studies reported in the literature.

Since Caspi et al. ${ }^{12}$ firstly evaluated GxE interactions between stressful life events and the 5-HTTLPR variant in the SLC6A4 gene contributing to depression, there have been more and more variants found to be associated with depression via complicated GxE interactions. The 5-HTTLPR variant in the SLC6A4 gene is normally represented as short and long alleles, where the short and long alleles are associated with lower and higher SLC6A4 gene expression activity, respectively. ${ }^{13,14}$ While the short allele in the 5-HTTLPR variant possesses 14 repeats of a sequence, the long allele possesses 16 repeats. Intriguingly, Caspi et al. ${ }^{12}$ found GxE interactions between the 5-HTTL$\mathrm{PR}$ variant and stressful life events to influence the risk for developing depression among subjects having one or both short alleles of the 5-HTTLPR variant in a prospective longitudinal study of Dunedin children $(n=1,037)$. Caspi et al. ${ }^{12}$ interviewed the individuals to assess their stressful life events appearing between 21 and 26 years old at regular intervals. Subsequently, Roy et al. ${ }^{15}$ reported that GxE interactions between the 5-HTTLPR variant and childhood trauma is associated with the tendency of suicidal behaviors for patients with a low-expressing 5-HTTLPR genotype; however, patients with intermediate-expressing and high-expressing genotypes did not have this tendency. Furthermore, Cicchetti et al. ${ }^{16}$ revealed evidence for GxE interactions between the 5-HTTLPR variant and various subtypes of maltreatment, where children of short/short or short/long genotypes in the 5-HTTLPR variant expressed higher suicidal risk than those of a long/long genotype in terms of children who had experienced one or two types of maltreatment. However, children who had experienced three to four types of maltreatment expressed higher levels of suicidal risk, disregarding any forms of genotypes in the 5-HTTLPR variant. ${ }^{16}$

The proposed candidate genes such as FKBP5, ${ }^{17-19}$ CRHR1, ${ }^{20,21}$ and $H T R 2 A^{22}$ have been assessed to verify the hypothesis that these loci could contribute to depression via complex GxE interactions. Appel et al. ${ }^{17}$ suggested GxE interactions between the FKBP5 rs1360780 SNP and physical abuse for developing depression in the German population. Similarly, Zimmermann et al. ${ }^{23}$ found that GxE interactions between the FKBP5 rs1360780 SNP and traumatic life events were linked to the onset of major depression in the UK population. Subsequently, Kohrt et al. ${ }^{18}$ confirmed that GxE interactions between the FKBP5 rs9296158 SNP and childhood maltreatment were associated with the risk of developing depression among South Asia adults. The FKBP5 gene encodes a protein that plays an important role in immunoregulation, where several 
SNPs in the FKBP5 gene have been found to be associated with depression. ${ }^{24}$ In the African Americans population, Roy et al. ${ }^{21}$ observed GxE interactions between the FKBP5 gene (such as rs9296158, rs3800373, and rs1360780 SNPs) and childhood trauma on suicide attempt by using the Childhood Trauma Questionnaire (CTQ) score. By using the continuous CTQ score, Roy et al. ${ }^{21}$ also revealed possible GxE interactions between three SNPs (such as rs7728378, rs6453267, and rs10474485 SNPs) in the CRHBP gene and childhood trauma in predicting suicide attempts.

Additionally, Ben-Efraim et al. ${ }^{20}$ revealed three GxE interactions for three subsets of distinct male patients in a cohort of a family-based study design where both parents and offspring had attempted suicide. In Ben-Efraim et al.'s ${ }^{20}$ results, one GxE interaction was only linked to female patients. BenEfraim et al. ${ }^{20}$ also reported significant $\mathrm{GxE}$ interactions between the CRHR1 rs7209436 SNP and childhood/adolescence physical attack/assault for both male and female patients with suicide attempts. In addition, significant $\mathrm{GxE}$ interactions involving the CRHR1 rs16940665 SNP and adulthood physical attack/assault were only suggested in male patients with suicide attempts. ${ }^{20}$ Moreover, GxE interactions between the CRHR1 rs4792887 SNP and cumulative stressful life events in male patients with suicide attempts were shown to support the previous studies. ${ }^{20}$

In a subsequent study, Ben-Efraim et al. ${ }^{22}$ revealed potential GxE interactions between the rs6313 SNP in the HTR2A gene and cumulative types of lifetime stressful life events in a family-based study design of both parents and offspring $(n=660)$ who had committed a suicide attempt. In addition, Ben-Efraim et al. ${ }^{22}$ reported significant $\mathrm{GxE}$ interactions involving the rs7322347 SNP in the HTR2A gene and childhood/adolescence physical assault for female patients. The HTR2A gene encodes one of the serotonin receptors and has been indicated as a potential marker for antidepressant therapy and psychiatric diseases such as major depressive disorders, anxiety disorders, eating disorders, attention deficit hyperactivity disorders, obsessive-compulsive disorders, Alzheimer's disease, and schizophrenia. ${ }^{25,26}$

In a recent meta-analysis study, Culverhouse et al. ${ }^{27}$ did not confirm that GxE interactions between stressful life events and 5-HTTLPR can contribute to the development of depression by using 38,802 subjects of European ancestry. Culverhouse et al. $\mathrm{s}^{27}$ analysis suggests that the interaction influencing depression is not commonly generalizable. While the hypothesis-driven candidate gene approach can only explore a fraction of probable existing genetic variants, ${ }^{28}$ a hypothesisfree approach such as GWAS has not been able to generalize the loci including FKBP5, CRHR1, and HTR2A so far.

In a GxE GWAS study, Dunn et al. ${ }^{29}$ investigated GxE inter- actions between stressful life events and identified a significant GxE interaction among the rs4652467 SNP near the CEP350 gene and history of childhood trauma in influencing the risk of depression in the African American population ( $n \sim 10,000$ ). Intriguingly, the rs4652467 variant reached genome-wide significant threshold. In another GxE GWAS study, Otowa et al. ${ }^{30}$ found a GxE interaction involving the rs10510057 SNP near the RGS10 gene and history of stressful events in influencing the increased risk of depression in the Japanese population $(n=320)$. However, the rs10510057 variant did not pass genome-wide significant threshold. In a recent GxE GWAS study, Van der Auwera et al. ${ }^{31}$ did not replicate these two variants (rs4652467 and rs10510057) and did not discover any novel genes in subjects of European ancestry $(n=3,944)$, suggesting that the inconsistent results may be due to the different phenotype definition, populations of different ancestry, and small sample size. While it is a theoretically feasible choice to employ GWAS approach to evaluate GxE interactions, GWAS would require huge test datasets as well as replication datasets with both exposure (such as stressful life events) and outcome (such as depression) data. ${ }^{32}$

To narrow the number of potential candidate genes, Cattaneo et al. ${ }^{32}$ proposed to use cross-species and cross-tissue biological prioritization approaches and identified three novel genes, including $A 2 M$, FoxO1, and TGF- $\beta 1$, that have GxE interactions with stressful life events (such as childhood trauma) contributing to the development of depression. Cattaneo et $\mathrm{al}^{32}$ pinpointed 16 genes (including $A 2 M, A R I D 5 B, A R R D C 4$, EPHA4, FBXO32, FoxO1, HSF2, ISOC1, LDLRAP1, LRRN3, MYO1D, PIK3C2B, PPAP2A, SAMD12, SERINC5, and TGF$\beta 1)$ and then identified a minimal set of genes that have potential gene-gene interactions among these 16 genes and thereby may belong to common or overlapping signaling systems. The minimal set consists of three genes such as A2M, FoxO1, and TGF- $\beta 1$ involved in cytokines signaling, TGF- $\beta 1$ signaling, and glucocorticoid receptor signaling. Subsequently, these three genes were utilized for $\mathrm{GxE}$ analysis to show significant GxE interactions with stressful life events, such as childhood sexual, physical or emotional abuse, contributing to the development of depression. ${ }^{32}$

\section{Epigenetic mechanisms and depression}

In light of recent advances in scientific research, evidence indicates that epigenetic mechanisms such as DNA methylation, microRNAs, and histone modifications play a key role in psychiatric diseases such as depression. ${ }^{33}$

DNA methylation is a process that involves the addition of a methyl group to the DNA molecule, particularly when a cytosine is succeeded by a guanine (CpG dinucleotide).$^{34}$ Increasing evidence implicates that DNA methylation, a prima- 
ry epigenetic factor, is universally linked to lower transcriptional activity, where DNA methylation is modulated by a family of DNA methyl-transferase proteins. ${ }^{35}$ While most investigations on DNA methylation have mainly pinpointed CpG islands (such as $1 \mathrm{~kb}$ CpG-rich regions) in promoter regions, DNA methylation in other genomic regions (such as intergenic regions or gene bodies) remains to be elucidated..$^{35}$

Moreover, small noncoding RNAs, particularly microRNA, represent a new arena in epigenetics research. MicroRNAs are less than 200 nucleotides in length. ${ }^{36,37}$ Noncoding RNAs, such as microRNA, consist of a sequence of nucleotides that does not encode proteins.

Furthermore, histone proteins thoroughly cooperate with DNA to form nucleosomes. ${ }^{33}$ A histone modification, such as histone acetylation or histone deacetylation, is a covalent posttranslational modification to histone proteins.

We focus on the latest developments in the field of epigenetics with respect to depression. A growing amount of research studies have been conducted when investigators remain to pay much attention to epigenetics research. This review is not intended as a comprehensive survey of all possible epigenetic reports studied in the literature.

\section{DNA methylation}

It has been implicated that the epigenetic mechanism of DNA methylation is associated with childhood maltreatment in both animal and human studies. ${ }^{38}$ Current evidence also demonstrates that stressful events during childhood and adulthood have an impact on the increased risk of psychiatric disorders such as depression. ${ }^{38}$ Numerous studies with a candidate-gene approach suggested that the epigenetic mechanism of DNA methylation in various genes, such as BDNF, NR3C1, OXTR, and SLC6A4, is associated with depression. ${ }^{9,39-41} \mathrm{Fu}-$ chikami et al. ${ }^{42}$ found that DNA methylation of the BDNF gene can be used as a biomarker for differentiating healthy controls from patients with depression due to different methylation levels of $\mathrm{CpG}$ units within CpG I of the BDNF gene between healthy controls and patients with depression.

In an animal study, Roth et $\mathrm{al}^{43}$ demonstrated that elevated DNA methylation within the $B d n f$ gene is associated with stressful early-life events for rats by using an animal model of childhood maltreatment. In accordance with Roth et al.s ${ }^{43}$ results, subsequent animal studies verified that the $B d n f$ gene is associated with alterations in DNA methylation. ${ }^{44,45}$ It should be noted that the BDNF gene is a well-known potential marker for playing a role in the pathophysiology of depression in both animal and human studies. ${ }^{46}$ In the context of animal models, it has also been implicated that stressful early-life events can regulate the DNA methylation level of genes, such as the Avp, Esr1, Gad1, Gdnf, Nr3c1, and Slc6a4 genes, in ani- mal studies. ${ }^{47-52}$

In addition, Tyrka et al. ${ }^{53}$ demonstrated that reduced methylation levels of the NR3C1 gene were linked to childhood maltreatment and depressive disorders in human adults ( $\mathrm{n}=$ 340). Na et al. ${ }^{54}$ also showed that hypomethylation of the NR3C1 gene in blood samples was linked to patients of depression $(n=45)$, when matched to healthy controls $(n=72)$. It has been suggested that declined hippocampal glucocorticoid receptor expression was linked to depression and suicide, where the NR3C1 gene encodes glucocorticoid receptor. ${ }^{55}$ In a postmortem hippocampus study, McGowan et al. ${ }^{56}$ explored whether epigenetic differences exist in the promoter region of the NR3C1 gene by matching suicide victims $(n=12)$ with a history of childhood abuse to healthy controls $(n=12)$. McGowan et al. $s^{56}$ analysis detected elevated cytosine methylation in abused suicide victims at two discrete $\mathrm{CpG}$ regions in the promoter site of the NR3C1 gene, hypothesizing that DNA methylation may prevail into adulthood. In accordance with McGowan et al. ${ }^{56}$ study, Weaver et al. ${ }^{57}$ observed that epigenetic alterations in the $\mathrm{Nr} 3 \mathrm{cl}$ gene is modulated by early life events in an animal study of epigenetic regulation in related genomic sites.

Given that BDNF-NTRK2 signature has been linked to depression and suicide, Ernst et al. ${ }^{58}$ investigated whether NTRK2 expression is significantly reduced in suicide patients when matched to healthy controls and whether methylation is linked to the downregulation. In a postmortem case-control study, Ernst et al..$^{58}$ evaluated microarray data to test the hypothesis by using HG-U133 chips (Affymetrix, High Wycombe, England). Ernst et al. ${ }^{58}$ reported the downregulated expression of the NTRK2 gene in suicide completers and observed that this downregulation was modulated by the methylation state at two specific $\mathrm{CpG}$ dinucleotides of the promoter site in the NTRK2 gene in the frontal cortex of suicide completers. It has been suggested that a truncated polymorphism of the NTRK2 gene is expressed in astrocytes.

In a methylation-sensitive restriction enzyme-based study, Sabunciyan et al. ${ }^{59}$ tested the genome-wide DNA methylation scan with a microarray platform (containing 3.5 million CpGs) by matching postmortem frontal cortex samples $(n=39)$ of depressive patients to healthy controls $(n=26)$. Sabunciyan et al. ${ }^{59}$ observed the greatest difference in the PRIMA1 gene with $12-15 \%$ elevated DNA methylation in depressive patients; however, this observation did not remain significant after multiple testing correction. Because DNA methylation alterations are unlikely to occur in the frontal cortex of depressive patients, the major target might be in other brain regions such as hippocampus and amygdala. ${ }^{59}$

Furthermore, several epigenome-wide association studies (EWAS) have been conducted to identify the probable interrelationship between DNA methylation and depression. ${ }^{59-63}$ In 
an EWAS study, Uddin et al. ${ }^{62}$ assessed genome-wide methylation profiles of about 14,000 genes and demonstrated different DNA methylation patterns between patients with a lifetime history of depression $(n=33)$ and healthy controls $(n=67)$ by using methylation microarrays. In another EWAS study, Numata et al. ${ }^{61}$ assessed genome-wide methylation profiles of 431,489 CpG sites and found lower DNA methylation at 363 CpG sites in medication-free patients with depression $(n=20)$ compared to healthy controls $(n=19)$ by using methylation microarrays, suggesting that the DNA methylation biomarkers can be used to distinguish patients with depression from healthy controls with good performance.

Moreover, Byrne et al. ${ }^{60}$ reported lower global DNA methylation levels in female patients with depression, but not among male subjects, in an Australian EWAS study of 24 monozygotic twin pairs discordant for depression. Sabunciyan et al. ${ }^{59}$ also identified hypermethylation of the PRIMA1 gene in a pilot EWAS study; however, this result could not be confirmed in the replication data. Finally, Tseng et al. ${ }^{63}$ detected that there was a decrease in global DNA methylation in older patients with severe depression, but not in younger patients, in a Taiwanese EWAS study.

\section{MicroRNAs}

Maffioletti et al. ${ }^{64}$ identified 5 microRNAs (including hsalet-7a-5p, hsa-let-7d-5p, hsa-let-7f-5p, hsa-miR-24-3p, and hsa-miR-425-3p) that were specifically altered in the blood of depressive patients, suggesting dysregulated microRNAs likely modulate the expression of brain-related genes. In order to pinpoint presumed microRNA signals associated with depression, Maffioletti et al. ${ }^{64}$ leveraged a microarray technique, which has the expression levels of 1,733 mature microRNAs, and used the blood of 20 depressive patients and 20 healthy controls. Maffioletti et al. ${ }^{64}$ findings indicated that microRNAs play a key role in the pathogenesis of depression.

In a genome-wide gene expression study, Garbett et al. ${ }^{65}$ explored whether transcriptome-based profiles can be employed as peripheral markers for depression by using dermal fibroblasts from depressive patients. Garbett et al. ${ }^{65}$ assayed dermal fibroblast samples $(n=32)$ from both depressive patients and healthy controls and utilized a genome-wide mRNA expression approach by using GeneChip HT HG-U133+ PM Array Plate (Affymetrix, USA). In order to assess an interaction involving the mRNA and microRNA expression alterations, Garbett et al. ${ }^{65}$ carried out quantitative polymerase chain reaction-based analysis of microRNA species. By comparing the fibroblasts of depressive patients to healthy controls, Garbett et al ${ }^{65}$ reported a potent mRNA gene expression alteration in varied molecular pathways for cell-to-cell communication (including the MET, PCDH10, PPL, and TNXB genes). Addition- ally, Garbett et al. ${ }^{65}$ showed hsa-miR-122, the most prominent microRNA candidate, significantly expressing in the hippocampus. Moreover, Garbett et al. ${ }^{65}$ suggested that the mRNA and microRNA expression alterations naturally collaborated with each other.

Similarly, Li et al. ${ }^{66}$ investigated the aspects of the BDNF protein and BDNF-relevant microRNAs in the pathogenesis of depression. By comparing depressive patients $(n=40)$ with healthy controls $(n=40)$, Li et al's ${ }^{66}$ study revealed that there were decreased serum BDNF levels as well as greater serum miR-132 and miR-182 levels. In addition, Lopez et al. ${ }^{67}$ identified miR-1202 that targets the expression of the GRM4 gene, which was up-regulated in patients with depression. Fan et al. ${ }^{68}$ also reported that 5 miRNAs (including miRNA-26b, miRNA-1972, miRNA-4485, miRNA-4498, and miRNA-4743) were up-regulated in patients with depression.

\section{Histone modifications}

In general, histone acetylation is directly linked to active chromatin and metabolic production of acetyl coenzyme A. ${ }^{69,70}$ In a variety of models, various studies have examined that histone acetylation in the hippocampus might play a longterm adaptive role in stress and antidepressant responses. ${ }^{71-73}$ In an animal study, Covington et al. ${ }^{74}$ pinpointed histone acetylation to be transiently reduced and then regularly attained in the nucleus accumbens, an important limbic brain site, after chronic social defeat stress. Covington et al.'s ${ }^{74}$ results implicate that histone acetylation might play a key role in both stress and depression. In addition, Hunter et al. ${ }^{75}$ reported regulation of histone methylation concerning chronic stress in the hippocampus, suggesting that there is an interaction between stress and hippocampal gene expression. Moreover, there are some novel treatment options based on histone deacetylase inhibitors for depression, especially for treatment-resistant depression by utilizing the antidepressant-like efficacy of histone deacetylase inhibitors. ${ }^{76,77}$

\section{DISCUSSION}

It should be pointed that that the aforementioned studies are faced with some limitations. Firstly, the smaller-scale sample size of the cohort warrants no well-clarified discoveries. ${ }^{78}$ Secondly, because various variants and markers were not replicated well among independent datasets, it makes us to wonder whether the novel findings are well-evaluated. ${ }^{79}$ Thirdly, it is crucial to probe possible variants and markers among numerous ethnic populations due to the fact that various populations may draw distinctive conclusions. ${ }^{37}$

In order to weigh $\mathrm{GxE}$ interactions, future studies might take advantage of utilizing new artificial intelligence and machine 
learning techniques such as deep learning artificial neural network algorithms. ${ }^{80,81}$ In order to assess GxE interactions, feasible artificial intelligence and machine learning algorithms encompass a wide spectrum of models such as artificial neural networks, Bayesian networks, decision trees, generative adversarial networks, support vector machines, and regression models. ${ }^{82}$ Furthermore, future research can contribute to identify genetic and epigenetic markers by using whole genome sequencing ${ }^{83}$ or exome sequencing. ${ }^{84}$ Whole genome sequencing serves as an overall approach in genomic research and provides a wide variety of genetic variants in an individual subject due to the reduced cost and expanded throughput from next-generation sequencing techniques. ${ }^{85}$ Exome sequencing, which selectively sequences the nucleotides of protein-coding exons in an individual subject, has been employed as an alternative and efficient approach for Mendelian disorders and common diseases. ${ }^{84}$ All in all, combining whole genome sequencing or exome sequencing with innovative artificial intelligence and machine learning algorithms might likely accomplish a comprehensive understanding of GxE interactions in depression in future research.

In future work, artificial intelligence and machine learning pipelines can be used to provide a thorough validation and evaluate whether we are able to replicate the current findings in predictive and diagnostic research studies. Additionally, we should explore possible genetic and epigenetic markers by utilizing custom artificial intelligence and machine learning pipelines; thereby, genetic and epigenetic networks would be interpreted at the genome level. In order to precisely understand pathogenesis and therapy in depression, future work must ultimately figure out how to integrate multiple markers and multi-omics, such as clinical data, genetics, transcriptomics, metabolomics, proteomics, epigenetics, and imaging data. ${ }^{79}$ In additional, artificial intelligence and machine learning techniques (such as deep learning, computer vision, and natural language processing) may play a pivotal role in eliminating the false positive candidate variants and genes that were observed in the previous association studies with meta-analysis, GxE interaction analysis, epigenetic analysis, and pathway models. ${ }^{82}$ Artificial intelligence and machine learning models involving with multi-omics data not only will achieve better results when dealing with incomplete data from any single data source, but also will bridge the gap among various phenotypes, genomic mechanisms, and biological regulation models. ${ }^{86}$ Although forecast testing for disease status and treatment responses in depression are now nonexistent ahead of diagnosis, it is anticipated that artificial intelligence and machine learning approaches will be leveraged to predict the tendency of drug efficacy and to contribute meaningful guidance for clinicians on determining personalized medications in future research. ${ }^{87}$

\section{Acknowledgments}

This study was supported in part by grants from the Ministry of Science and Technology of Taiwan (grant MOST 107-2634-F-075-002) and from the Taipei Veterans General Hospital (grant V105D17-002-MY2-2). The author thanks Emily Ting for English editing. The authors declare that they have no conflicts of interest in writing this report.

\section{Conflicts of Interest}

The authors have no potential conflicts of interest to disclose.

\section{Author Contributions}

Conceptualization: Eugene Lin, Shih-Jen Tsai. Funding acquisition: ShihJen Tsai. Investigation: Eugene Lin, Shih-Jen Tsai. Methodology: Eugene Lin. Project administration: Shih-Jen Tsai. Resources: Eugene Lin, Shih-Jen Tsai. Supervision: Shih-Jen Tsai. Writing_original draft: Eugene Lin. Writing_review \& editing: Eugene Lin, Shih-Jen Tsai.

\section{ORCID iDs}

Shih-Jen Tsai https://orcid.org/0000-0002-9987-022X

Eugene Lin https://orcid.org/0000-0001-6984-2159

\section{REFERENCES}

1. Lin E, Tsai SJ. Gene-Environment Interactions and Role of Epigenetics in Depression. In: Kim YK, Editor. Understanding Depression. Singapore: Springer, 2018, p.41-50.

2. Peña CJ, Nestler EJ. Progress in Epigenetics of Depression. In: Grayson DR, Editor. Progress in Molecular Biology and Translational Science. Amsterdam: Elsevier, 2018, p.41-66.

3. Howard DM, Adams MJ, Shirali M, Clarke TK, Marioni RE, Davies G, et al. Genome-wide association study of depression phenotypes in UK Biobank identifies variants in excitatory synaptic pathways. Nat Commun 2018;9:1470.

4. Hyde CL, Nagle MW, Tian C, Chen X, Paciga SA, Wendland JR, et al. Identification of 15 genetic loci associated with risk of major depression in individuals of European descent. Nat Genet 2016;48:1031-1036.

5. Wray NR, Ripke S, Mattheisen M, Trzaskowski M, Byrne EM, Abdellaoui A, et al. Genome-wide association analyses identify 44 risk variants and refine the genetic architecture of major depression. Nat Genet 2018;50:668-681.

6. Howard DM, Adams MJ, Clarke TK, Hafferty JD, Gibson J, Shirali M, et al. Genome-wide meta-analysis of depression identifies 102 independent variants and highlights the importance of the prefrontal brain regions. Nature Neurosci 2019;22:343-352.

7. Menke A, Klengel T, Binder EB. Epigenetics, depression and antidepressant treatment. Curr Pharm Des 2012;18:5879-5889.

8. Nestler EJ. Epigenetic mechanisms of depression. JAMA Psychiatry 2014; 71:454-456.

9. Dalton VS, Kolshus E, McLoughlin DM. Epigenetics and depression: return of the repressed. J Affect Disord 2014;155:1-12.

10. Shapero BG, Black SK, Liu RT, Klugman J, Bender RE, Abramson LY, et al. Stressful life events and depression symptoms: the effect of childhood emotional abuse on stress reactivity. J Clin Psychol 2014;70:209-223.

11. Sharma S, Powers A, Bradley B, Ressler KJ. Gene x environment determinants of stress- and anxiety-related disorders. Ann Rev Psychol 2016; 67:239-261.

12. Caspi A, Sugden K, Moffitt TE, Taylor A, Craig IW, Harrington H, et al. Influence of life stress on depression: moderation by a polymorphism in the 5-HTT gene. Science 2003;301:386-389.

13. Gibb BE, McGeary JE, Beevers CG, Miller IW. Serotonin transporter (5-HTTLPR) genotype, childhood abuse, and suicide attempts in adult psychiatric inpatients. Suicide Life Threat Behav 2006;36:687-693.

14. Lin E, Tsai SJ. Genetics and Suicide. In: Courtet P, Editor. Understanding Suicide. Cham: Springer, 2016, p.85-95. 
15. Roy A, Hu XZ, Janal MN, Goldman D. Interaction between childhood trauma and serotonin transporter gene variation in suicide. Neuropsychopharmacology 2007;32:2046-2052.

16. Cicchetti D, Rogosch FA, Sturge-Apple M, Toth SL. Interaction of child maltreatment and 5-HTT polymorphisms: suicidal ideation among children from low-SES backgrounds. J Pediatr Psychol 2009;35:536-546.

17. Appel K, Schwahn C, Mahler J, Schulz A, Spitzer C, Fenske K, et al. Moderation of adult depression by a polymorphism in the FKBP5 gene and childhood physical abuse in the general population. Neuropsychopharmacology 2011;36:1982-1991.

18. Kohrt BA, Worthman CM, Ressler KJ, Mercer KB, Upadhaya N, Koirala S, et al. Cross-cultural gene-environment interactions in depression, post-traumatic stress disorder, and the cortisol awakening response: FKBP5 polymorphisms and childhood trauma in South Asia: GxE interactions in South Asia. Int Rev Psychiatry 2015;27:180-196.

19. Roy A, Gorodetsky E, Yuan Q, Goldman D, Enoch MA. Interaction of FKBP5, a stress-related gene, with childhood trauma increases the risk for attempting suicide. Neuropsychopharmacology 2010;35:1674-1683.

20. Ben-Efraim Y, Wasserman D, Wasserman J, Sokolowski M. Gene-environment interactions between CRHR1 variants and physical assault in suicide attempts. Genes Brain Behav 2011;10:663-672.

21. Roy A, Hodgkinson CA, DeLuca V, Goldman D, Enoch MA. Two HPA axis genes, CRHBP and FKBP5, interact with childhood trauma to increase the risk for suicidal behavior. J Psychiatr Res 2012;46:72-79.

22. Ben-Efraim Y, Wasserman D, Wasserman J, Sokolowski M. Family-based study of HTR2A in suicide attempts: observed gene, genexenvironment and parent-of-origin associations. Mol Psychiatry 2013;18:758-766.

23. Zimmermann P, Brückl T, Nocon A, Pfister H, Binder EB, Uhr M, et al. Interaction of FKBP5 gene variants and adverse life events in predicting depression onset: results from a 10-year prospective community study. Am J Psychiatry 2011;168:1107-1116.

24. Szczepankiewicz A, Leszczyńska-Rodziewicz A, Pawlak J, Narozna B, Rajewska-Rager A, Wilkosc M, et al. FKBP5 polymorphism is associated with major depression but not with bipolar disorder. J Affect Disord 2014;164:33-37.

25. Lane HY, Tsai GE, Lin E. Assessing gene-gene interactions in pharmacogenomics. Mol Diagn Ther 2012;16:15-27.

26. Lin E, Chen PS. Pharmacogenomics with antidepressants in the STAR* D study. Pharmacogenomics 2008;9:935-946.

27. Culverhouse RC, Saccone NL, Horton AC, Ma Y, Anstey KJ, Banaschews$\mathrm{ki} \mathrm{T}$, et al. Collaborative meta-analysis finds no evidence of a strong interaction between stress and 5-HTTLPR genotype contributing to the development of depression. Mol Psychiatry 2018;23:133-142.

28. Heim C, Binder EB. Current research trends in early life stress and depression: review of human studies on sensitive periods, gene-environment interactions, and epigenetics. Exp Neurol 2012;233:102-111.

29. Dunn EC, Wiste A, Radmanesh F, Almli LM, Gogarten SM, Sofer T, et al. Genome-wide association study (GWAS) and genome-wide by environment interaction study (GWEIS) of depressive symptoms in African American and Hispanic/Latina women. Depress Anxiety 2016;33:265280

30. Otowa T, Kawamura Y, Tsutsumi A, Kawakami N, Kan C, Shimada T, et al. The first pilot genome-wide gene-environment study of depression in the Japanese population. PLoS one 2016;11:e0160823.

31. Van der Auwera S, Peyrot WJ, Milaneschi Y, Hertel J, Baune B, Breen G, et al. Genome-wide gene-environment interaction in depression: a systematic evaluation of candidate genes: the childhood trauma workinggroup of PGC-MDD. Am J Med Genet B Neuropsychiatr Genet 2018; 177:40-49.

32. Cattaneo A, Cattane N, Malpighi C, Czamara D, Suarez A, Mariani N, et al. FoxO1, A2M, and TGF- $\beta 1$ : three novel genes predicting depression in gene $\mathrm{X}$ environment interactions are identified using cross-species and cross-tissues transcriptomic and miRNomic analyses. Mol Psychiatry 2018;23:2192-2208.

33. Peña CJ, Bagot RC, Labonté B, Nestler EJ. Epigenetic signaling in psy- chiatric disorders. J Mol Biol 2014;426:3389-3412.

34. Klose RJ, Bird AP. Genomic DNA methylation: the mark and its mediators. Trends Biochem Sci 2006;31:89-97.

35. Jones PA. Functions of DNA methylation: islands, start sites, gene bodies and beyond. Nat Rev Genet 2012;13:484-492.

36. Nagano T, Fraser P. No-nonsense functions for long noncoding RNAs. Cell 2011;145:178-181.

37. Lin E, Tsai SJ. Genome-wide microarray analysis of gene expression profiling in major depression and antidepressant therapy. Prog Neuropsychopharmacol Biol Psychiatry 2016;64:334-340.

38. Lutz PE, Turecki G. DNA methylation and childhood maltreatment: from animal models to human studies. Neuroscience 2014;264:142-156.

39. Bakusic J, Schaufeli W, Claes S, Godderis L. Stress, burnout and depression: A systematic review on DNA methylation mechanisms. J Psychosom Res 2017;92:34-44.

40. Li M, D'Arcy C, Li X, Zhang T, Joober R, Meng X. What do DNA methylation studies tell us about depression? A systematic review. Transl Psychiatry 2019;9:68.

41. Lockwood LE, Su S, Youssef NA. The role of epigenetics in depression and suicide: A platform for gene-environment interactions. Psychiatry Res 2015;228:235-242.

42. Fuchikami M, Morinobu S, Segawa M, Okamoto Y, Yamawaki S, Ozaki $\mathrm{N}$, et al. DNA methylation profiles of the brain-derived neurotrophic factor (BDNF) gene as a potent diagnostic biomarker in major depression. PLoS One 2011;6:e23881.

43. Roth TL, Lubin FD, Funk AJ, Sweatt JD. Lasting epigenetic influence of early-life adversity on the BDNF gene. Biol Psychiatry 2009;65:760-769.

44. Blaze J, Asok A, Roth TL. Long-term effects of early-life caregiving experiences on brain-derived neurotrophic factor histone acetylation in the adult rat mPFC. Stress 2015;18:607-615.

45. Doherty TS, Forster A, Roth TL. Global and gene-specific DNA methylation alterations in the adolescent amygdala and hippocampus in an animal model of caregiver maltreatment. Behav Brain Res 2016;298:5561.

46. Hashimoto K. Brain-derived neurotrophic factor as a biomarker for mood disorders: an historical overview and future directions. Psychiatry Clin Neurosci 2010;64:341-357.

47. Champagne FA, Weaver IC, Diorio J, Dymov S, Szyf M, Meaney MJ. Maternal care associated with methylation of the estrogen receptor- $\alpha 1 b$ promoter and estrogen receptor- $\alpha$ expression in the medial preoptic area of female offspring. Endocrinology 2006;147:2909-2915.

48. Kinnally EL, Capitanio JP, Leibel R, Deng L, LeDuc C, Haghighi F, et al. Epigenetic regulation of serotonin transporter expression and behavior in infant rhesus macaques. Genes Brain Behav 2010;9:575-582.

49. Murgatroyd C, Patchev AV, Wu Y, Micale V, Bockmühl Y, Fischer D, et al. Dynamic DNA methylation programs persistent adverse effects of early-life stress. Nature Neuroscience 2009;12:1559-1566.

50. Uchida S, Hara K, Kobayashi A, Otsuki K, Yamagata H, Hobara T, et al. Epigenetic status of Gdnf in the ventral striatum determines susceptibility and adaptation to daily stressful events. Neuron 2011;69:359-372.

51. Weaver IC, Meaney MJ, Szyf M. Maternal care effects on the hippocampal transcriptome and anxiety-mediated behaviors in the offspring that are reversible in adulthood. Proc Natl Acad Sci U S A 2006;103:3480-3485.

52. Zhang TY, Hellstrom IC, Bagot RC, Wen X, Diorio J, Meaney MJ. Maternal care and DNA methylation of a glutamic acid decarboxylase 1 promoter in rat hippocampus. J Neurosci 2010;30:13130-13137.

53. Tyrka AR, Parade SH, Welch ES, Ridout KK, Price LH, Marsit C, et al. Methylation of the leukocyte glucocorticoid receptor gene promoter in adults: associations with early adversity and depressive, anxiety and substance-use disorders. Transl Psychiatry 2016;6:e848.

54. Na KS, Chang HS, Won E, Han KM, Choi S, Tae WS, et al. Association between glucocorticoid receptor methylation and hippocampal subfields in major depressive disorder. PLoS One 2014;9:e85425.

55. Webster M, Knable M, O'grady J, Orthmann J, Weickert CS. Regional specificity of brain glucocorticoid receptor mRNA alterations in subjects 
with schizophrenia and mood disorders. Mol Psychiatry 2002;7:985-994, 924.

56. McGowan PO, Sasaki A, D’alessio AC, Dymov S, Labonté B, Szyf M, et al. Epigenetic regulation of the glucocorticoid receptor in human brain associates with childhood abuse. Nature Neurosci 2009;12:342-348.

57. Weaver IC, D’Alessio AC, Brown SE, Hellstrom IC, Dymov S, Sharma S, et al. The transcription factor nerve growth factor-inducible protein a mediates epigenetic programming: altering epigenetic marks by immediate-early genes. J Neurosci 2007;27:1756-1768.

58. Ernst C, Deleva V, Deng X, Sequeira A, Pomarenski A, Klempan T, et al. Alternative splicing, methylation state, and expression profile of tropomyosin-related kinase B in the frontal cortex of suicide completers. Arch Gen Psychiatry 2009;66:22-32.

59. Sabunciyan S, Aryee MJ, Irizarry RA, Rongione M, Webster MJ, Kaufman WE, et al. Genome-wide DNA methylation scan in major depressive disorder. PLoS One 2012;7:e34451.

60. Byrne E, Carrillo-Roa T, Henders A, Bowdler L, McRae A, Heath A, et al. Monozygotic twins affected with major depressive disorder have greater variance in methylation than their unaffected co-twin. Transl Psychiatry 2013;3:e269.

61. Numata S, Ishii K, Tajima A, Iga J, Kinoshita M, Watanabe S, et al. Blood diagnostic biomarkers for major depressive disorder using multiplex DNA methylation profiles: discovery and validation. Epigenetics 2015; 10:135-141.

62. Uddin M, Koenen K, Aiello A, Wildman D, de Los Santos R, Galea S. Epigenetic and inflammatory marker profiles associated with depression in a community-based epidemiologic sample. Psychol Med 2011; 41:997-1007.

63. Tseng PT, Lin PY, Lee Y, Hung CF, Lung FW, Chen CS, et al. Age-associated decrease in global DNA methylation in patients with major depression. Neuropsychiatr Dis Treat 2014;10:2105-2114.

64. Maffioletti E, Cattaneo A, Rosso G, Maina G, Maj C, Gennarelli M, et al. Peripheral whole blood microRNA alterations in major depression and bipolar disorder. J Affect Disord 2016;200:250-258.

65. Garbett KA, Vereczkei A, Kálmán S, Brown JA, Taylor WD, Faludi G, et al. Coordinated messenger RNA/microRNA changes in fibroblasts of patients with major depression. Biol Psychiatry 2015;77:256-265.

66. Li YJ, Xu M, Gao ZH, Wang YQ, Yue Z, Zhang YX, et al. Alterations of serum levels of BDNF-related miRNAs in patients with depression. PLoS One 2013;8:e63648.

67. Lopez JP, Lim R, Cruceanu C, Crapper L, Fasano C, Labonte B, et al. miR1202 is a primate-specific and brain-enriched microRNA involved in major depression and antidepressant treatment. Nat Med 2014;20:764-768.

68. Fan HM, Sun XY, Guo W, Zhong AF, Niu W, Zhao L, et al. Differential expression of microRNA in peripheral blood mononuclear cells as specific biomarker for major depressive disorder patients. J Psychiatr Res 2014;59:45-52.

69. Mews P, Donahue G, Drake AM, Luczak V, Abel T, Berger SL. AcetylCoA synthetase regulates histone acetylation and hippocampal memory. Nature 2017;546:381-386

70. Pradeepa MM, Grimes GR, Kumar Y, Olley G, Taylor GC, Schneider R, et al. Histone $\mathrm{H} 3$ globular domain acetylation identifies a new class of enhancers. Nature Genet 2016;48:681-686.
71. Covington III HE, Vialou VF, LaPlant Q, Ohnishi YN, Nestler EJ. Hippocampal-dependent antidepressant-like activity of histone deacetylase inhibition. Neurosci Lett 2011;493:122-126.

72. Hollis F, Duclot F, Gunjan A, Kabbaj M. Individual differences in the effect of social defeat on anhedonia and histone acetylation in the rat hippocampus. Horm Behav 2011;59:331-337.

73. Sun H, Kennedy PJ, Nestler EJ. Epigenetics of the depressed brain: role of histone acetylation and methylation. Neuropsychopharmacology 2013; 38:124-137.

74. Covington HE, Maze I, LaPlant QC, Vialou VF, Ohnishi YN, Berton O, et al. Antidepressant actions of histone deacetylase inhibitors. J Neurosci 2009;29:11451-11460.

75. Hunter RG, McCarthy KJ, Milne TA, Pfaff DW, McEwen BS. Regulation of hippocampal $\mathrm{H} 3$ histone methylation by acute and chronic stress. Proc Natl Acad Sci U S A 2009;106:20912-20917.

76. Deussing JM, Jakovcevski M. Histone Modifications in Major Depressive Disorder and Related Rodent Models. In: Delgado-Morales R, Editor. Neuroepigenomics in Aging and Disease. Cham: Springer, 2017, p. 169-183.

77. Fuchikami M, Yamamoto S, Morinobu S, Okada S, Yamawaki Y, Yamawaki S. The potential use of histone deacetylase inhibitors in the treatment of depression. Prog Neuropsychopharmacol Biol Psychiatry 2016; 64:320-324.

78. Lin E, Lane HY. Genome-wide association studies in pharmacogenomics of antidepressants. Pharmacogenomics 2015;16:555-566.

79. Lin E, Tsai S. Machine Learning and Predictive Algorithms for Personalized Medicine: From Physiology to Treatment. New York: Personalized Medicine Nova Science Publishers; 2016.

80. Lin E, Hong CJ, Hwang JP, Liou YJ, Yang CH, Cheng D, et al. Gene-gene interactions of the brain-derived neurotrophic-factor and neurotrophic tyrosine kinase receptor 2 genes in geriatric depression. Rejuvenation Res 2009;12:387-393.

81. Lou XY, Chen GB, Yan L, Ma JZ, Zhu J, Elston RC, et al. A generalized combinatorial approach for detecting gene-by-gene and gene-by-environment interactions with application to nicotine dependence. Am J Hum Genet 2007;80:1125-1137.

82. Lin E, Lane HY. Machine learning and systems genomics approaches for multi-omics data. Biomark Res 2017;5:2.

83. Cirulli ET, Goldstein DB. Uncovering the roles of rare variants in common disease through whole-genome sequencing. Nat Rev Genet 2010; 11:415-425.

84. Bamshad MJ, Ng SB, Bigham AW, Tabor HK, Emond MJ, Nickerson $\mathrm{DA}$, et al. Exome sequencing as a tool for Mendelian disease gene discovery. Nat Rev Genet 2011; 12: 745-755.

85. Hodkinson BP, Grice EA. Next-generation sequencing: a review of technologies and tools for wound microbiome research. Adv Wound Care (New Rochelle) 2015;4:50-58.

86. Lin E, Kuo PH, Liu YL, Yu YW, Yang A, Tsai SJ. A deep learning approach for predicting antidepressant response in major depression using clinical and genetic biomarkers. Front Psychiatry 2018;9:290.

87. Lin E, Lin CH, Lai YL, Huang CH, Huang YJ, Lane HY. Combination of G72 genetic variation and G72 protein level to detect schizophrenia: machine learning approaches. Front Psychiatry 2018;9:566. 